VIP + neurons

support, whereas

upregulated

SST + neuronal

activity

impairs, the

maintenance

of action plans

in the dmPFC

\title{
Keeping short-term memories alive
}

If a response to a stimulus has to be delayed during a task, neural activity in the prefrontal cortex (PFC) is thought to maintain task-relevant information as a short-term memory. However, the circuit basis of this so-called delay activity is not clear. In a new study in mice, Kamigaki and Dan shed light on the contributions of different cell types in the dorsomedial PFC (dmPFC) to such activity.

The authors trained mice on a delay-response 'go-no-go' task. In this task, animals were presented with a 'go' stimulus (an $8 \mathrm{kHz}$ tone) or a 'no-go' stimulus (a $2 \mathrm{kHz}$ tone) and then waited for a delay period of 5 seconds. After the delay, the mice were rewarded with water for licking on 'go' trials and were punished for licking on 'no-go' trials (termed 'false alarm' responses).

Calcium imaging of dmPFC pyramidal neurons during this task revealed that some neurons were more active during the delay in go trials, whereas other neurons were more active during the delay on no-go trials. During the first 2 seconds of the delay period, optogenetic activation of somatostatin-expressing $\left(\mathrm{SST}^{+}\right.$) interneurons and parvalbuminexpressing $\left(\mathrm{PV}^{+}\right)$interneurons, which inhibit cortical pyramidal cells, decreased task performance, mainly by increasing the rate of false alarms. Together, these data suggest that PFC pyramidal cells maintain action plan information during the delay and are particularly important for suppressing false alarms.
A third major group of interneurons in the dmPFC expresses vasoactive intestinal peptide $\left(\mathrm{VIP}^{+}\right.$ interneurons), and previous research has shown that these interneurons disinhibit pyramidal cells by inhibiting $\mathrm{SST}^{+}$and $\mathrm{PV}^{+}$neurons. Here, optogenetic activation or inhibition of $\mathrm{dmPFC} \mathrm{VIP}{ }^{+}$interneurons (either for the whole trial or for the first 2 seconds of the delay) improved or impaired task performance, respectively, through changes in false alarm rate - thus, $\mathrm{VIP}^{+}$neuron activity may also be required to suppress false alarm responses.

Interestingly, optogenetic activation of dmPFC VIP ${ }^{+}$interneurons suppressed the activity of go-preferring pyramidal neurons on no-go trials (but not on go trials) and the activity of no-go-preferring pyramidal neurons on go trials (but not no-go trials). Thus, $\mathrm{VIP}^{+}$neuron activity can differentially modulate the activity of pyramidal neurons depending on the type of trial and the preference (or function) of the pyramidal neuron.

To investigate the roles of $\mathrm{SST}^{+}$and $\mathrm{VIP}^{+}$neurons in memory-guided behaviour that did not involve response suppression, the authors trained mice on a task in which, after the presentation of a $2 \mathrm{kHz}$ or $8 \mathrm{kHz}$ tone and subsequent variable delay, mice had to lick either the left or the right of two water ports, respectively. As in the go-no-go task, optogenetic activation of $\mathrm{SST}^{+}$ neurons impaired task performance, whereas similar activation of $\mathrm{VIP}^{+}$ neurons improved performance. Thus, VIP ${ }^{+}$neurons support, whereas upregulated $\mathrm{SST}^{+}$neuronal activity impairs, the maintenance of action plans in the dmPFC.

Together, these results reveal how these three interneuron populations affect the maintenance of short-term memory in the dmPFC. The authors point out that $\mathrm{VIP}^{+}$neurons receive many inputs from other cortical areas, and thus modulation of working memory could be mediated by $\mathrm{VIP}^{+}$neurons.

Natasha Bray

ORIGINAL ARTICLE Kamigaki, T. \& Dan, Y. Delay activity of specific prefrontal interneuron subtypes modulates memory-guided behavior. Nat. Neurosci. http://dx.doi.org/10.1038/nn.4554 (2017)

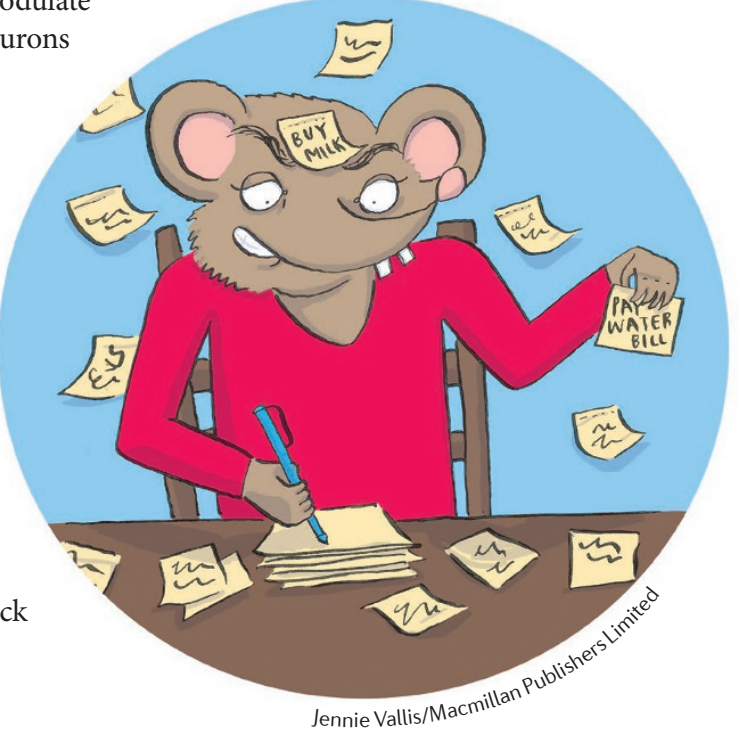

